peripheral neuropathy. 2 months later a thigh mass developed. Biopsy showed peripheral T-cell lymphoma, CD3+, CD4+, CD5+, CD56+, PD-1+, T-cell intracellular antigen-1 (TIA-1)+ and TCR-beta+, and CD30-, TdT-, CD34-, CD138-, and CD21-. Rare cells were $\mathrm{CD} 8+$ and $\mathrm{CD} 20+$, and positive for TCR gamma gene rearrangement by $\mathrm{PCR}$. The sural nerve biopsy showed additional immunopositivity for CD4, CD56, and TIA-1, matching the thigh mass lymphoma immunophenotype.

Conclusions: T-cell lymphoma of peripheral nerves has been described as a solitary mass of the median nerve and in the presence of widely disseminated lymphoma. We demonstrate for the first time the utility of aberrant CD56 and TIA-1 immunopositivity in diagnosing lymphomatous neuropathy. This case also draws attention to several other reports of apparently benign lymphocytic infiltrates affecting nerves in systemic non-Hodgkin lymphomas may in fact represent lymphomatous involvement of nerve.

\section{Ophthalmic Pathology}

1823 Clinicopathologic Features of Orbital Space-Occupying Lymphoid Lesions: A Single Institution Experience

$J$ Boone, F Fan. University of Kansas Medical Center, Kansas City, KS.

Background: Lymphoid-rich lesions compose a large proportion of space-occupying lesions of the orbit. Differential diagnoses include infectious, inflammatory/reactive, autoimmune and neoplastic processes. It poses diagnostic challenge to pathologists due to limited biopsy material and often times overlapping histologic features. Current literature on this subject is comprised of mostly case reports. This study is designed to collect clinicopathological data on lymphoid space occupying lesions of the orbit in our hospital

Design: A list of patients diagnosed with a lymphoid infiltrative lesion of the orbit was obtained from the surgical pathology specimen records. The reports were reviewed and histologic data including diagnosis, immunohistochemical staining, flow cytometry, and molecular testing were recorded. The cases were divided into four categories as: reactive/inflammatoy, IgG4 related sclerosing dacryoadenitis, atypical lymphoid infiltrate and lymphoma. The critical ancillary test that led to the final diagnosis was identified. Pertinent clinical information were also collected.

Results: There were a total of 44 cases over an eleven-year period in our institution. The majority of cases $(88.6 \%)$ represented a primary disease process in the orbital region. Male to female ratio was 2:1 and the age range was 31-98 years old. Among the 44 cases, 22 cases were reactive/inflammatory $(50 \%), 2$ were IgG4 related disease $(4.5 \%), 6$ were atypical lymphoid infiltrate $(13.6 \%)$, and 14 were lymphoma $(31.8 \%)$. All lymphoma cases were B-cell lymphomas with extranodal marginal zone lymphoma being the most common (57.1\%) type, followed by follicular lymphoma, diffuse large B-cell lymphoma and mantle cell lymphoma. Ancillary testing (immunohistochemical staining, flow cytometry and gene rearrangement) was used in $81 \%$ of cases to reach a final diagnosis. Among them, flow cytometry was identified to be the critical ancillary test most frequently.

Conclusions: Orbital space-occupying lymphoid lesions are rare overall. Most are primary orbital lesions with half being benign reactive/inflammatory process. The second most common lesion is B-cell lymphoma. IgG4 related sclerosing disease makes only a small portion of cases. Flow cytometry appears to be the most useful test in the work-up of orbital lymphoid lesions and should be considered a priority in cases with limited tissue size.

1824 Clinicopathologic Correlations in 2,487 Consecutive Corneal Specimens from a Single Ophthalmic Pathology Laboratory YJ Cruz-Inigo, DO Hodge, JJ Garcia, SV Patel, DR Salomao. Mayo Clinic, Rochester, $\mathrm{MN}$.

Background: Corneal specimens are unfamiliar to a general surgical pathologist. Biopsies are performed to exclude infectious processes and resections to treat generalized corneal diseases and correct optical consequences. We reviewed clinical and pathological records of all corneal specimens submitted to our laboratory to determine differences between intramural (in-house), extramural (consultation) specimens, and trends over time.

Design: Retrospective search of surgical pathology database for all corneal specimens submitted from January 1994 to July 2012. Reports were evaluated for patients' demographics, clinical indications, procedure type and histopathological interpretation. Statistical analysis was performed.

Results: Study cohort comprised of 2,487 specimens from 2,011 patients: 887 males (44\%) and 1,124 females (56\%); mean age 64 yrs (median $70 \mathrm{yrs}$; range 2 mos- $102 \mathrm{yrs)}$ ); $50 \%$ from right, $47 \%$ from left, and $3 \%$ undesignated. Most were intramural specimens (61\%); penetrating (73\%) or lamellar keratoplasties (19\%), and corneal biopsies $(9 \%)$. Multiple specimens on a single patient were intramural cases only (24\%). Statistical analysis showed percentage of intramural and extramural penetrating keratoplasties decreased $(p<0.001)$ and lamellar keratoplasties increased $(p<0.001)$ over time. Percentage of corneal biopsies, intramural $(p=0.36)$ and extramural $(p=0.16)$, remained constant. Most frequent pathologic diagnoses were Fuchs' dystrophy (27\%), bullous keratopathy $(18 \%)$, keratoconus $(16 \%)$, graft failure $(10 \%)$ and corneal scarring $(9 \%)$. Comparison between intramural and extramural cases revealed that Fuchs' dystrophy ( $33 \%$ vs $17 \% ; p<0.001)$ and corneal ulceration $(6 \%$ vs $3 \% ; p=0.003)$ were more often diagnosed in intramural specimens, while graft failure $(13 \%$ vs $19 \% ; p<0.001)$ and degenerations ( $1 \%$ vs $3 \% ; p<0.001)$ were more often in extramural specimens. On trend analysis, the percentage of intramural procedures performed for Fuchs' dystrophy $(p=0.007)$ and graft failure $(p<0.001)$ increased over time; same was observed for graft failure $(p=0.008)$ and scarring $(p=0.002)$ on extramural specimens. Bullous keratopathy decreased over time in the latter group $(p<0.001)$.
Conclusions: Our study confirms a trend in surgical treatment of corneal diseases; lamellar keratoplasty is becoming more common in both intramural and extramural practices. In this series, most common diagnoses were Fuchs' dystrophy, bullous keratopathy, keratoconus and graft failure. The latter diagnosis has increased over time in both intramural and extramural specimens.

1825 Adenoid Cystic Carcinoma of the Lacrimal Gland Is Frequently Characterized by $\mathrm{t}(6 ; 9)$ and May Be Distinguished from Other Neoplasms Using Fluorescent In Situ Hybridization

MG Keeney, WR Sukov, JA Garrity, DR Salomao, JJ Garcia. Mayo Clinic, Rochester, $\mathrm{MN}$.

Background: Adenoid cystic carcinoma (ACC) of the lacrimal gland is a rare tumor that is almost universally fatal. ACC is composed of a dual cell population (ductal and myoepithelial cells) and may demonstrate tubular, cribriform, and/or solid architectural patterns. Unfortunately, these histopathologic features are not specific to ACC and can be seen in other lacrimal gland neoplasms, occasionally posing a diagnostic dilemma. Although $\mathrm{t}(6 ; 9)(\mathrm{q} 22-23 ; \mathrm{p} 23-24)$, involving $M Y B$ and $N F I B$, has been identified in a significant portion of ACC cases arising from other anatomic sites, its role in the pathogenesis of lacrimal gland ACC has not been elucidated. This study explores the incidence of $\mathrm{t}(6 ; 9)$ in ACC arising from the lacrimal gland, and the diagnostic utility of fluorescence in situ hybridization (FISH) in the clinical setting.

Design: Retrospective clinical and histopathologic review of 6 cases of lacrima gland ACC seen at Mayo Clinic over a 14-year period (1998-2012) was performed. Clinical data including patient age, sex, tumor size, treatment (surgery, radiotherapy, and/or chemotherapy), and clinical outcome parameters were obtained from medical records when available. Surgical pathology archival material was re-examined and formalin-fixed paraffin-embedded (FFPE) material was further evaluated with IHC when appropriate. FFPE material from all 6 cases and normal controls was assessed with FISH using a $M Y B$ break-apart probe designed and validated for clinical use. Results: The median patient age was 59.5 years (range $25-75$ ) and 4 patients were male (67\%). Rearrangement of $M Y B$ was identified using FISH in 3 cases $(50 \%)$ while normal controls showed no evidence of $M Y B$ rearrangement. No correlation between $M Y B$ rearrangement and histopathologic features, disease-specific, or overall survival was observed.

Conclusions: These study results show that $t(6 ; 9)$ is a frequent event in lacrimal gland ACC. The incidence of MYB rearrangement in lacrimal gland ACC rivals that of ACC arising from other anatomic sites - approximately $50 \%$. To this end, FISH for MYB rearrangement may be used as a diagnostic tool when the diagnosis of ACC of the lacrimal gland is in question. Lastly, the presence of $\mathrm{t}(6 ; 9)$ in ACC may provide a platform for molecular targeting strategies in the future.

\section{Investigation of C-MET in Uveal Melanoma}

SM Kennedy, C Barr, M Clynes, A Larkin. Royal Victoria Eye and Eye and Ear Hospital, Dublin 4, Ireland; National Institute for Cellular Biotechnology, Dublin City University, Dublin 9, Ireland.

Background: Uveal melanoma is the most common primary intraocular neoplasm affecting adults, over $50 \%$ of patients develop incurable metastatic disease. There is an urgent need to develop effective therapies for metastatic uveal melanoma. Altered c-MET signalling is known to be involved in uveal melanoma progesssion. The mechanism of cMET activation is unknown. Selective MET inhibition has been suggested as a potentially useful therapeutic target in uveal melanoma. Significantly lower BRAF mutation rates and increased rates in c-MET mutations have recently been reported in Irish patients with cutaneous melanoma (1). The aim of this retrospective study was to investigate c-MET activation in Irish patients diagnosed with uveal melanoma.

Design: Expression of cMET and activated phospho cMET was studied using Immuncytochemistry on TMAs from 192 enucleated tumours from Irish patients (86 female, 104 male) diagnosed with uveal melanoma with minimum 10 year follow-up. The study group included 78 spindle, 25 epitheloid and 84 mixed cell type tumours, $45 \%$ of this cohort developed metastasis and died of disease. cMET immunoreactivity was scored as zero, weak (1), intermediate (2) or strong (3) based on the intensity of the staining observed.

Results: Total cMET staining was predominantly cytoplasmic, with some weak membrane positivity observed. cMET was positive in $152(79 \%)$ of tumours, with strong staining observed in $21(10 \%)$, intermediate staining in $62(33 \%)$ and weak staining in $36 \%$ of tumours. Strong c-MET immunoreactivity was associated with epithelioid type tumours and a higher percentage of tumours which showed metastasis exhibited strong c-MET immunoreactivity compared to tumours without metastasis. Phospho cMET immunostaining has been optimised in cutaneous melanoma tumours, phospho cMET expression in this uveal melanoma patient cohort will be correlated with clinicopathological features of patients.

Conclusions: c-MET is highly expressed in Irish uveal melanoma patients; strong cMET immunoreactivity is associated with epithelioid tumour cell type and metastasis indicating that high c-MET expression is associated with adverse outcome. The results of this study will provide important information regarding the incidence of phospho activated cMET in Irish uveal melanoma patients, and will have implications for potential development of cMET as a potential therapeutic target for the treatment of metastatic uveal melanoma in the Irish population.

1 Gallagher WM, AACR 2013 (Abstract). 
1827 Human Papillomavirus in Ocular Squamous Lesions

PJ Kobalka, BS Korn, DO Kikkawa, JH Lin. University of California, San Diego, San Diego, CA; VA San Diego Healthcare System, San Diego, CA.

Background: Human Papillomavirus (HPV) is tightly associated with the development of cervical dysplasia and carcinoma, and more recently, HPV has been linked to squamous lesions at other sites, especially head and neck. The role of HPV in the development of squamous lesions of the eye is poorly understood and controversial. Here, we retrospectively surveyed squamous lesions identified in ocular biopsies, enucleations, and orbital exenterations performed at our institution and examined them for HPV infection via immunohistochemistry and PCR.

Design: The ocular lesions were first evaluated for $\mathrm{p} 16$ immunoreactivity since $\mathrm{p} 16$ is strongly induced by HPV infection. Cases with strong diffuse positivity for p16 were further assessed for HPV serotype expression by PCR genotyping (ARUP HPV panel). The squamous ocular lesions selected for this study included seborrheic keratoses (SKs), pterygiums, verruca vulgari (VVs), squamous papillomas, achrocordons, conjunctival intraepithelial neoplasms (CIN), and squamous cell carcinomas (SCCs). All lesions were reviewed histologically for additional features, including solar damage and dysplasia. The lesions were further subdivided by patient gender and age and analyzed for trends. Cases reviewed were from The University of California San Diego (UCSD) and VA San Diego Hospital in which tissue specimens were submitted to the eye pathology service. In total, we analyzed approximately 50 seborrheic keratoses, 30 pterygiums, 12 achrocordons, 10 CINs, 10 SCCs, 5 squamous papillomas, and 5 VVs. Results: As of this submission $85 \%$ of SKs (11 of 13) tested have shown p16 immunoreactivity; $67 \%$ of pterygiums ( 4 of 6 ) have been positive; $86 \%$ of CINs ( 6 of $7) ; 100 \%$ of VVs ( 2 of 2 ) and $100 \%$ of SCCs ( 4 of 4 ). There was no clear link between cases that were p16-reactive and clinical demographics including age, gender, and ethnicity in our samples. The ocular squamous lesions further analyzed by HPV PCR genotyping were all negative for high-risk subtypes.

Conclusions: Our findings revealed a surprisingly large prevalence of p16 immunoreactivity in nearly all squamous lesions at our institutions. No clear correlation was seen between the p16-positive ocular squamous lesions and patient age or gender or ethnicity. Surprisingly, these lesions were not positive for high-risk HPV variants. Our findings raise the possibility that other HPV variants may be involved in development of ocular squamous lesions.

1828 Evaluation of Surgical Margins for Treatment of Ocular Surface Squamous Neoplasia (OSSN)

L Schoenfield, S Pabon, A Singh. Ohio State University Wexner Medical Center, Columbus, OH; Case Western Reserve University, Cleveland, OH; Cleveland Clinic Foundation, Cleveland, $\mathrm{OH}$.

Background: Ocular surface squamous neoplasia (OSSN) encompasses squamous dysplasia (mild-severe, CIN 1-3) and invasive squamous cell carcinoma (SCC) involving the conjunctiva and cornea. A traditionally accepted management modality is widemargin (4-5 $\mathrm{mm}$ ) excisional biopsy followed by cryotherapy. The conjunctival defect is then closed using conjunctival or amniotic membrane graft. Additional treatment strategies include chemotherapy and immunotherapy either in conjunction with or in lieu of surgery. Removing more tissue than necessary can compromise ocular function, for example by causing limbal cell insufficiency. Thus a re-evaluation was deemed warranted

Design: With IRB approval, a retrospective analysis of all patients presenting to one clinician (AS) with clinical OSSN from 2004-2012 requiring surgical excision was conducted.

Results: There were 32 cases, two of whom had been treated previously with topical chemotherapy for chemoreduction. All lesions were excised with $1-2 \mathrm{~mm}$ clinical margins and treated with cryotherapy. The mean follow-up time was 24.4 months (range: 1-84 months). In 17 cases there was corneal involvement. Pathological distribution of the lesions included 25 non-invasive OSSN ( 1 with CIN1, 5 CIN2, and 19 CIN3 or severe dysplasia/squamous carcinoma in situ) and 7 invasive SCC. 11 cases (34\%) had positive pathological margins. At 1-year and 5-year follow-up, only 2 of 32 eyes $(6 \%)$ had evidence of recurrence, respectively. The cases had SCC in situ and microinvasive SCC; both had positive initial margins. 3 patients in the cohort expired from causes unrelated to ocular disease.

Conclusions: OSSN can be managed surgically with adjunct cryotherapy using smaller margins without compromising clinical outcome. This may improve anatomical results and minimize the morbidity of the treatment.

1829 DLX Homeobox Transcription Factor Regulation of the Brn3a Homeobox Gene during Vertebrate Retina Development

$Q$ Zhang, D Eisenstat. Western University, London, ON, Canada; University of Alberta, Edmonton, AB, Canada.

Background: Retinal degeneration and retinal malignancy account for the majority of vision loss diseases. Our current knowledge of the genes involved in the processes of neuroretinal proliferation and cell differentiation is limited. The $D l \times 1 / D l \times 2$ double knockout mouse has reduced numbers ( $33 \%$ fewer) of retinal ganglion cells (RGC), due to enhanced apoptosis. Brn $3 a$ and Brn $3 b$ are closely related members of the Class IV POU-domain gene family, and share similar expression patterns and play functionally interchangeable roles in retinal development. $B r n 3 b$ is transcriptionally activated by both DLX1 and DLX2. We hypothesized that Brn3a is a DLX transcriptional target during retinal development.

Design: Embryonic retinas were dissected and chromatin immunoprecipitation (ChIP) of this tissue was utilized to identify DLX proteins bound to specific DNA elements of the Brn3a gene. Electrophoretic mobility shift assays (EMSA) were used to confirm specificity of this binding. Luciferase reporter gene assays were performed to confirm functional effects of DLX binding on the Brn $3 a$ promoter. In utero retinal electroporation was used as an in vivo functional study of DLX on Brn3a expression.

Results: Only DLX2 but not DLX1 protein bound to the Brn 3 a promoter in vivo. Using EMSA, recombinant DLX2 bound to specific Brn $3 a$ promoter sequences and specific supershifted bands resulted from the addition of DLX2 antibody. DLX2 binding to the Brn $3 a$ promoter region activated transcription of a luciferase reporter gene in vitro. In utero retinal electroporation shows that ectopic DLX2 expression promotes Brn $3 a$ expression in vivo.

Conclusions: DLX2, but not DLX1, regulates Brn $3 a$ transcription by directly binding to the Brn $3 a$ promoter region.

1830 Unsuspected Epithelial Neoplasias Associated with Pterygium: A 20 Year Study

P Zoroquiain, F Ceballos, H Dietrich, P Logan, VBravo-Filho, MN Burnier, Jr.. McGill University, Montreal, QC, Canada.

Background: Pinguecula and pterygium $(\mathrm{p} / \mathrm{pt})$ are conjunctival lesions strongly related to sunlight exposure. The incidence of these lesions varies considerably according to geographic areas. Ocular surface squamous neoplasia(OSSN) represents a broad spectrum of lesions starting as dysplasia and evolving to invasive squamous cell carcinoma of the conjunctiva. The relationship between $\mathrm{p} / \mathrm{pt}$ and OSSN has been described for various geographical areas including Brisbane, Australia (9.8\%; very high UV index), Florida, USA (1.7\%; moderate to high UV index), and in Ontario, Canada $(0 \%$; low UV index). To the best of our knowledge this is the first study looking at a Quebec patient population(low-UV index).

Design: All cases between 1993 and 2013 with a clinical diagnosis of $\mathrm{p} / \mathrm{pt}$ from the Henry C. Witelson Ocular Pathology Lab, McGill University, Montreal were reviewed. The final pathological diagnosis and clinical information, including age and gender, were obtained. Epithelial neoplasias were classified as actinic keratosis(a precancerous lesion), conjunctival intra epithelial neoplasia with mild dysplasia(CIN I), moderate dysplasia(CIN II), severe dysplasia/carcinoma in Situ(CIN III), and invasive squamous cell, mucoepidermoid, or sebaceous carcinoma.

Results: Two hundred and five cases were diagnosed clinically as pterygium and 22 as pinguecula; $51.2 \%$ of patients were male and $48.8 \%$ were female. The average age at diagnosis was $53.4 \pm 15.54$. The incidence of epithelial neoplasia in these $\mathrm{p} / \mathrm{pt}$ cases was $6.65 \% ; 60 \%$ were female and $40 \%$ were male and the average age was $53.8 \pm 14.9$. When analysed separately the incidence of epithelial neoplasia in pterygium and pinguecula were $4.88 \%$ and $18,88 \%$ respectively. The average age were $53.07 \pm 15.24$ and $58.75 \pm 21,47$ respectively. The average age of the patients with epithelial neoplasia associated to $\mathrm{p} / \mathrm{pt}$ is similar to the patients without neoplasia $(\mathrm{p}>0.05)$. The same results are found when pterygium is analysed separately. Epithelial neoplasias in $\mathrm{p} / \mathrm{pt}$ were diagnosed as actinic keratosis $(6.67 \%)$, CIN I (66.67\%), CIN II (13.33\%), CIN III $(13.33 \%)$

Conclusions: In this series, most epithelial lesions associated with p/pt were CIN I. The relative high proportion of neoplasia in pinguecula in this study could be due to the fact that pingueculas are surgically removed only when they have an abnormal appearance. Caution is advised due to the relative high prevalence of neoplastic lesions even in low UV index areas. Our relatively high rate of dysplasia in a low prevalence disease area with a low UV index challenges the main cause of this disease. This finding should be evaluated in new studies.

\section{Pancreas and Biliary Tree}

$1831 \quad$ Mutational Profiling (MP) of Discarded Supernatant Fluid: Its Role in Enhancing the Diagnostic Accuracy of Pancreatic Fine Needle Aspirations (FNA)

GAlsaati, S Nguyen, N Haddad, MK Sidawy. MedStar Georgetown University Hospital, Washington, DC

Background: Endoscopic ultrasound-guided (EUS) FNA of pancreatic lesions is associated with false negative results. Ancillary and molecular tests are emerging as useful tools for improving diagnostic accuracy. The purpose of this study was to evaluate the role of MP in discarded supernatant fluid from FNA of solid pancreatic lesions with negative, non-diagnostic (ND) or atypical cytologic diagnoses.

Design: Our database was searched (July 2011-July 2013) for EUS-FNA of solid pancreatic lesions which had supernatant fluid submitted for possible MP. 110 FNA from 108 patients were identified. 74 (67\%) were diagnostic of pancreatic neoplasms and the remaining 36 were classified as negative, ND or atypical. Only 21 of these 36 cases had available surgical or clinical follow-up and were included in the study. A clinically validated panel that included KRAS oncogene and 16 microsatellite markers associated with pancreaticobiliary cancer was used for MP. The results were classified as positive or negative for malignancy based on the presence of at least one mutation in any of the markers

Results: Table 1 summarizes the FNA, MP and follow-up findings. FNA diagnoses were: 8 negative, $10 \mathrm{ND}$ and 3 atypical. Follow-up revealed 6 pancreatic carcinomas and 15 benign outcomes. The supernatant of all 21 patients had sufficient amounts of amplifiable DNA for analysis. MP was positive in 2/6 malignant cases (one ND and one atypical by FNA). All cases with negative outcomes showed no mutations. 